\title{
La fin de Lost : jonction du temps et discours intemporel
}

\section{Vladimir Lifschutz}

\section{(2) OpenEdition}

1 Journals

Édition électronique

URL : http://journals.openedition.org/tvseries/1724

DOI : 10.4000/tvseries. 1724

ISSN : 2266-0909

Éditeur

GRIC - Groupe de recherche Identités et Cultures

Référence électronique

Vladimir Lifschutz, «La fin de Lost : jonction du temps et discours intemporel », TV/Series [En ligne], Hors séries 1 | 2016, mis en ligne le 01 octobre 2016, consulté le 05 mai 2019. URL : http:// journals.openedition.org/tvseries/1724; DOI : 10.4000/tvseries.1724

Ce document a été généré automatiquement le 5 mai 2019.

\section{(c) (i) (2) $\Theta$}

TV/Series est mis à disposition selon les termes de la licence Creative Commons Attribution - Pas d'Utilisation Commerciale - Pas de Modification 4.0 International. 


\title{
La fin de Lost : jonction du temps et discours intemporel
}

\author{
Vladimir Lifschutz
}

1 La simple évocation de la fin de Lost capte généralement l'attention de l'audience. Nous avons personnellement commencé à regarder Lost à l'âge de seize ans. Nous nous souvenons très bien des premiers épisodes diffusés sur TF1 à partir de l'été 2005. Nous avons alors pensé «Et bien! Ils ont intérêt à savoir où ils vont». Si la décision nous incombait, nous aurions aimé que la série se termine dès la première saison puisque nous voulions savoir si les rescapés de du vol Océanic 815 allaient réussir à quitter l'île. Nous désirions une réponse à la question posée par Charlie dès la fin du pilote de la série : « Les gars, où est-ce qu'on est?». Pourtant, à mesure que notre impatience était mise à l'épreuve, nous regardions Lost avec plus de détachement. Nous commencions à accepter l'idée que cela pouvait durer encore plusieurs années - avant que la décision d'achever la fiction lors de sa sixième saison fixe un nouvel horizon d'attente. À partir de ce moment, nous pouvions redevenir patiemment impatient puisque nous avions la certitude que notre attente serait contentée d'une manière ou d'une autre. En préparant ce travail, c'est le souvenir de tous ces sentiments qui nous est revenu. Nous avons tous notre propre histoire avec Lost. Chacun a bâti ce que nous pourrions appeler un « mode de regard » sur cette fiction. Cet historique personnel est nécessairement inscrit dans une temporalité. Que vous ayez suivi Lost lors de sa diffusion (en parallèle de la diffusion américaine sur Internet, voire sur les chaînes françaises) ou que vous ayez consommé la fiction en DVD, en téléchargement ou en VOD sur un laps de temps plus ou moins court, nous avons chacun eu notre expérience de cette fiction. C'est ce que nous pourrions appeler le temps "spectatoriel », c'est-à-dire la mémoire du temps que nous avons partagé avec la fiction et ce que nous en avons retiré. La plus grande spécificité de cette série me semble résider dans son articulation si particulière du temps. Un temps du souvenir, de l'anticipation, de l'alternance, un temps constructeur d'identité et d'affect, un temps de partage et de discussion. 
2 D'un point de plus pragmatique, chaque saison de Lost s'articule autour de deux trajectoires temporelles différentes. Nous y reviendrons. En nous concentrant sur cette spécificité temporelle, nous verrons que la fin de Lost entend faire l'impossible jonction entre deux temporalités du récit et constituer un discours qui vise au dépassement de l'œuvre vers un avenir intemporel. Se souvenir de la fiction au-delà de sa première diffusion, faire de la fin l'instant pivot où la série se donne les possibilités de persister dans la mémoire commune. C'est donc à travers une certaine philosophie de la fin que nous pouvons essayer de comprendre le rôle de la clôture d'un "récit » aussi vaste et aussi particulier qu'un feuilleton sériel comme Lost. Nous ne nous intéresserons pas ici au débat qui agite la fin de la fiction; nous n'essaierons pas de porter un jugement qualitatif sur cette dernière mais nous concentrerons sur son fonctionnement dans son rapport au temps et aux téléspectateurs.

Qu'est-ce que la fin de Lost? Est-ce la dernière saison? Le dernier épisode ? Pour notre part, nous appelons la fin de Lost les dernières minutes du dernier épisode. Ce choix est dicté par notre approche des fins qui emprunte son agencement théorique à Paul Ricœur et à Frank Kermode. En effet, il nous semble que chaque conclusion passe par une rupture, un point de non-retour que Ricœur théorise sous le terme de passage de l' immanence du récit à l'imminence de la fin du récit. Cette idée forte dont il puise la source dans l'œuvre de Frank Kermode ${ }^{1}$ tend à instaurer que tout récit fonctionne sur l'idée d'une fin immanente, sans cesse remise à plus tard par la présence d'une "crise ", mais qu'un point de passage concomitant à la fin de la crise permet de passer à l'imminence de la fin du récit. C'est ce point de passage qui nous intéresse et les modalités qui en découlent: «La crise ne marque pas l'absence de toute fin, mais la conversion de la fin imminente en fin immanente ${ }^{2}$.» Toujours selon cette théorie, l'imminence fait ressentir la proximité de la fin. Le point de passage propre à Lost semble se situer lors de la rencontre entre Jack et son père. Cette ultime confrontation est la conclusion d'une longue quête initiée par le personnage. Ce dernier a toujours été à la recherche de sa figure paternelle, et ce, bien avant le décès de Christian Shephard. Une fois sur l'île, les « visions » de son père l'amènent à partir à la recherche du corps de celui-ci. L'anamnèse (ou perte de l'amnésie) constitue un des récits pivots de la dernière saison puisqu'il s'agit de retrouver la mémoire pragmatique et émotionnelle des événements sur l'île dans la temporalité des flash-sideways. Dans un effet-miroir récurrent, tous les personnages s'éveillent à leurs souvenirs sauf Jack qui reste amnésique jusqu'aux dernières minutes de la fiction. Lui qui est pourtant la première figure éveillée sur l'île est le dernier à s'éveiller lors de la fin de la fiction. Dans la logique de la sixième saison, l'éveil de tous les personnages amorce la fin du récit. L'anamnèse de Jack entraîne donc logiquement la fin de la fiction. C'est donc lors de cette ultime réunion père/fils que Jack dépasse son amnésie et s'éveille aux souvenirs de sa vie sur l'île. Notre point de non-retour est donc l'éveil de Jack qui constitue un effet réflexif qui induit le réveil des téléspectateurs à l'imminence de la fin de Lost. À partir de cet instant, nous avons pleinement conscience que la fin s'est définitivement mise en marche.

\section{« Déformulation » de la « formule »}

En suivant la logique de Ricœur, il nous apparait que le point de rupture d'une série peut entraîner ce que nous appelons une «déformulation» de la «formule ». Nous empruntons le terme «formule» à Jean-Pierre Esquenazi. La formule correspond au 
" cadre strict d'une série ${ }^{3}$ ", c'est-à-dire à son encadrement structurel, esthétique, sonore et temporel qui lui permet de garder, semaine après semaine, une identité propre et reconnaissable. Par exemple, dans Lost, les deux lignes temporelles coexistent et composent l'ensemble des épisodes de la série. L'une de ces lignes est constante tout au long des saisons, c'est le présent de l'île. L'autre ligne temporelle se définit par rapport à la première. Dans les trois premières saisons, ce sont des flashbacks. Par la suite, cela peut être des flashforwards et lors de la dernière saison, des flash-sideways. La " déformulation " suppose qu'il y ait une vraie rupture avec ce qui a été mis en place précédemment, que le cadre soit dépassé pour laisser place à un bouleversement des codes établis.

L'un des premiers exemples explicites concerne la partition sonore de ces derniers instants de la fiction. Ainsi, la fin de Lost repose sur une composition musicale de Michael Giacchino intitulé « Moving On » de 7 minutes 55 secondes, le morceau le plus long jamais composé pour la série. Cette composition synthétise plusieurs thèmes récurrents de Lost (dont «Life \& Death ", " There's no place like home », " Oceanic 815 », notamment) afin de façonner une partition qui se veut le reflet du dispositif sonore de la fiction. Elle envahit progressivement la dernière séquence de Lost au point de fonctionner comme une forme de vague musicale de plus en plus intense qui vient nourrir le montage, notamment d'un point de vue rythmique. En faisant ce qui n'a jamais était fait, Giacchino effectue sa révolution musicale par une synthèse sonore des thèmes récurrents de la fiction, entre hommage et nouveauté.

Dans un article intitulé "Fins de séries", les auteurs Laura Odello et Peter Szendy métaphorisent la fin comme « shutdowns » (« liquidation générale ») :

L'arrêt d'une série et l'arrêt du flux des liquidités [...] On pourrait le formuler ainsi : la série, en tant que succession de promesses de récit qui s'enchaînent dans une certaine surenchère cumulative est elle aussi entraînée dans une spirale de dette. Son enjeu, c'est en effet d'ouvrir une sorte de crédit narratologique croissant. Et dès lors, les fins des séries sont presque toujours des moments exemplaires où l'économie sérielle frôle le défaut de paiement et joue avec lui : comment clôturer tous les comptes, comment rembourser tous les emprunts contractés auprès des spectateurs, alors même que la logique de la capitalisation narrative vise plutôt à accroître les attentes, à relever sans cesse leur plafond, à ouvrir toujours plus de promesses ${ }^{4}$ ?

7 La métaphore nous semble intéressante puisqu'elle met en exergue la difficile conciliation d'une fin avec les attentes des téléspectateurs. L'idée de liquidation nous apparait pertinente puisqu'elle entraîne une forme de déraillement du système établi. L'idée de "déformulation" semble rejoindre cette conception. Le train sériel lancé à toute vitesse n'est pas ralenti mais est précipité vers son déraillement. Comment les auteurs de Lost ont-ils organisé ce moment de déraillement/liquidation/déformulation?

\section{There is no now here}

Le dialogue final entre Jack et son père joue un double rôle. Tout d'abord, un peu plus tôt, le contact avec le cercueil provoque l'anamnèse de Jack, le souvenir de son passé fictionnel. Sur la forme, cette prise de conscience est mise en scène par un montage qui réutilise des images de la fiction portées par un rythme dynamique et des images étalonnées pour accentuer les couleurs, ce qui produit un effet légèrement onirique. Celles-ci dévoilent le statut des flash-sideways, temporalité hors du temps, entre la vie et la 
mort, temps de l'anamnèse, temps créé par les protagonistes pour se retrouver et se souvenir. Cette ligne temporelle a donc une fonction qui est de «préparer les spectateurs à la fin de la série, de les aider à faire un travail de deuil et de rétrospection : se souvenir en flashback des moments marquants, se retourner sur le chemin parcouru, se rappeler ce que nous avons vécu depuis six ans ${ }^{5}$." Dans ce processus de "déformulation", le dialogue entre Jack et Christian marque un point de non-retour puisqu'il dépasse les frontières de la mort. Les paroles de Christian Shephard fonctionnent comme un double discours qui insiste sur ce point. "There is no now, here " ( Il n'y a pas de maintenant, ici ») pourrait se traduire, de manière méta-filmique, par « Tout ceci est de la fiction, ce n'est pas réel». «Everybody dies sometimes, kiddo» («Tout le monde meurt un jour, fiston ») serait l'équivalent de "Tout à une fin, même Lost ». "This is a place that you all made together so you can find one another " ( C'est un lieu que vous avez tous créé ensemble pour que vous puissiez vous retrouver »), sonne comme une explication de ce qu'est la série, un endroit de fiction créé avec l'assistance des spectateurs pour partager des histoires. "The most important part of your life was the time that you spent with these people, that's why all of you are here [...] you needed all of them and they needed you " (« Les moments les plus importants de ta vie, tu les as passés avec eux, c'est pour ça que vous êtes tous ici [...] Tu avais besoin d'eux et ils avaient besoin de toi ») apparaît comme l'explication du lien entre la fiction et les téléspectateurs: en tant qu'auteurs, l'aventure de Lost est l'expérience la plus importante de nos vies, nous avions besoin de vous et vous aviez besoin de nous. La théorisation de ce lien si particulier est poursuivie par le symbolique " To remember and let go » (« Pour se souvenir et lâcher prise ») qui vient accompagner les téléspectateurs dans leur travail de deuil pour qu'ils puissent se souvenir de la fiction et accepter sa finitude. Ceci est synthétisé par l'expression «Moving on» («passer à autre chose ») qui achève ce travail de rupture, comme si les auteurs confiaient: « Nous ne vous abandonnons pas, nous allons vers de nouveaux projets avec, nous l'espérons, votre fidélité. » La série ne marque pas seulement son achèvement par l'anamnèse de Jack et par la rencontre avec son père, elle l'énonce clairement, prenant les téléspectateurs par la main à l'instar du rôle de guide échu au personnage de Christian dans cette fin. Nous avons tous notre mode de regard et Christian s'adresse à toutes nos histoires; les auteurs utilisent les mots du père pour récapituler l'expérience de Lost pour eux-mêmes, pour les personnages et pour les téléspectateurs - triple discours inédit qui témoigne de la marque de la « déformulation ».

9 La question du temps est importante. Jack a besoin de savoir où il se trouve, mais Christian ne peut répondre à ce besoin, « il n'y a pas de maintenant, ici. » Le temps des flash-sideways est hors du temps. À l'inverse des flashbacks disséminés au cours des six saisons de la fiction ou des flashforwards présents à partir de la fin de la troisième saison, les flash-sideways n'appartiennent pas à la grande chronologie pragmatique de Lost. Là où les autres lignes temporelles prennent une place précise, le temps alternatif se veut un temps de l'imminence hors du temps. Nous pouvons néanmoins les situer en prenant en exemple deux séquences de Lost. La première est celle du décès de Juliette dans «LAX Part $1 »$ (S6E01). Juste avant de mourir, elle énonce quelques paroles étranges dont le sens est révélé lors du dernier épisode de Lost, « End Part II » (S6E18). Juliette n'était pas en train de divaguer; elle avait en quelque sorte déchiré les voiles temporels si bien qu'elle pouvait percevoir et réunir deux temporalités. L'anamnèse de Sawyer et Juliette permet de mettre en perspective cette jonction, le dialogue faisant écho aux propos de Juliette lors de sa mort. L'articulation de ce nouveau rapport au temps s'articule sur une 
compréhension qui demande aux spectateurs de réactualiser leur mémoire. Il est, en effet, impossible de comprendre ce nouveau rapport temporel sans avoir en tête les deux épisodes en question. L'autre séquence qui met à mal les frontières du temps est la dernière séquence de l'épisode final où Jack, proche de sa mort, perçoit l'autre temps.

\section{Jonction inédite du temps}

10 Pour la première fois, dans un même épisode, dans une même séquence, les deux lignes temporelles qui structurent l'ensemble de Lost se rejoignent. Ces deux trajectoires sont soumises à des variations tout au long de la fiction, mais elles ne se rejoignent jamais simultanément. Les flashbacks fonctionnement en général à la manière de ce que Gérard Genette appelle des analepses externes ${ }^{6}$ qu$^{\prime} i l$ définit comme en dehors du récit premier dans Lost, il s'agit des événements survenus avant le crash aérien qui marque l'entrée dans la fiction. En cela, il ne peut y avoir de jonction simultanée avec le récit premier. Les flashforwards fonctionnent, quant à eux, comme les prolepses de Genette: on peut distinguer les séquences qui sont finalement rejointes par le récit premier (prolepses complétives) et celles qui redoublent un segment narratif à venir (prolepse répétitive) ${ }^{7}$. Lors du renversement temporel par un flashforward lors du dernier épisode de la troisième saison, le récit se dédouble : on suit à la fois une histoire qui se déroule au présent et une histoire qui se passe dans l'avenir. Par la suite, le récit premier se met en quête de rejoindre ce nouveau segment narratif, ce qu'il parvient à faire lors du dernier épisode de la quatrième saison. Pourtant, la jonction ne se fait pas au même moment. Alors que le récit premier complète les flashforwards, ce dernier n'est pas immobile et continue d'avancer. Il y a donc des jonctions temporelles dans Lost, mais elles reposent sur une articulation complétive. Les jonctions ne mettent pas à mal les deux lignes temporelles puisqu'elles ne se rejoignent pas simultanément. Les deux lignes temporelles étant des forces toujours en action, il semble impossible de confondre ces deux lignes en une seule au même moment. C'est encore le cas lors de la dernière saison qui propose un temps présenté d'abord comme une ligne alternative des événements de la fiction.

11 Pourtant, la dernière séquence réussit l'impossible jonction inédite de deux lignes temporelles qui ont constamment joué au chat et à la souris tout au long de la fiction, cherchant une complétude qui semblait impossible jusqu'à cette dernière séquence. Par un jeu de montage alterné fondé sur des raccords de mouvement, les deux lignes temporelles se mettent à coexister. Jack prend conscience de sa double présence, à la fois sur l'île et dans l'église. 
Figures 1 et 2 : Montage final de Lost; la rencontre de deux temporalités, le présent de l'île et les flashsideways
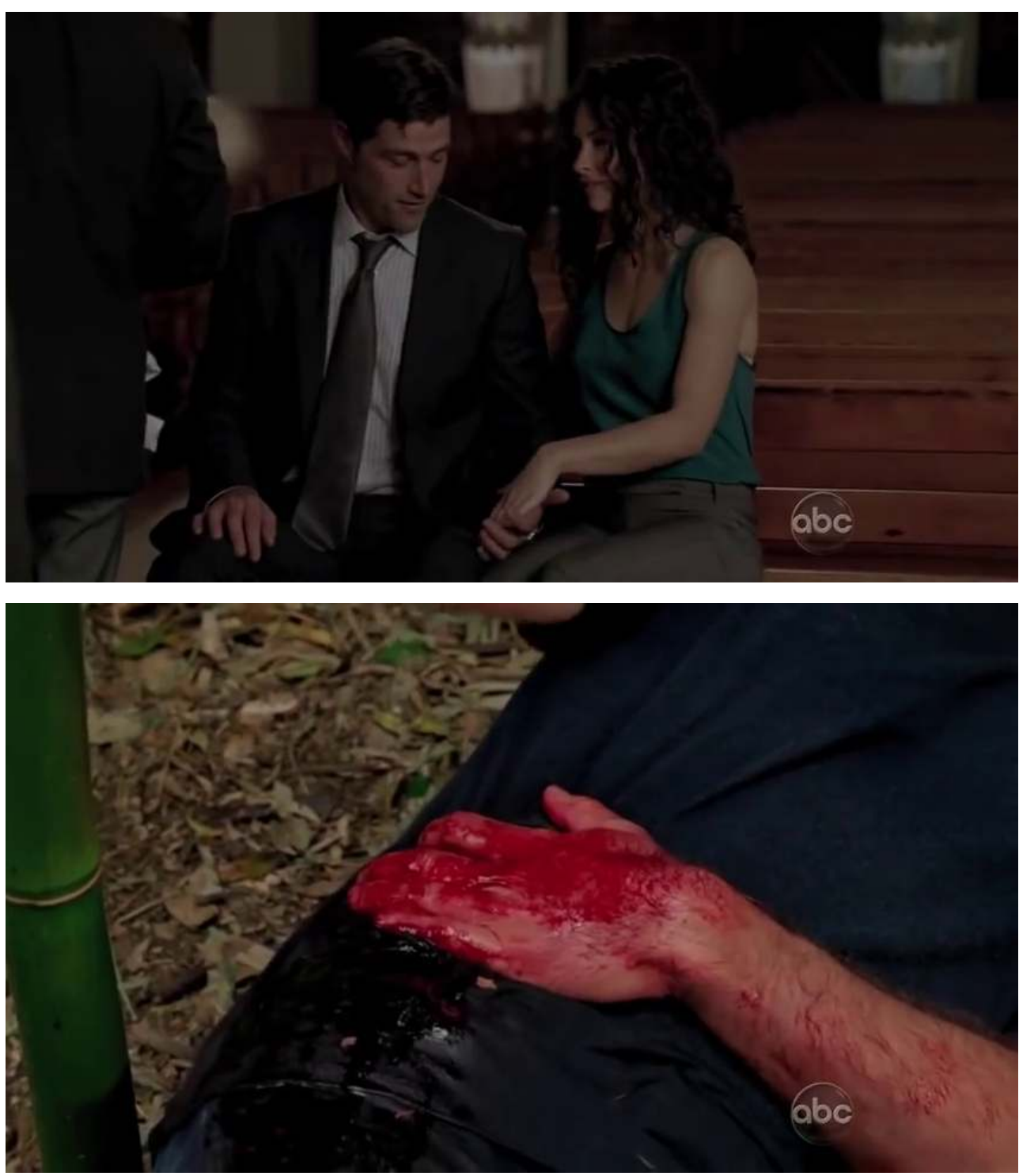

Grâce à au montage, les gestes deviennent les liants du temps. Jack s'écroule dans la forêt alors qu'il s'assoit à l'église ; la main de Kate posée sur celle de Jack fait écho au geste de Jack qui appuie sur sa blessure; la présence du chien renvoie à la figure du père ${ }^{8}$. Si le raccord-mouvement assure la cohérence du montage, le regard reste l'élément qui fusionne les temps. L'œil dévoile les deux lignes temporelles et permet à Jack de vivre l'imminence de sa fin sous la forme d'une double énonciation temporelle. Bien évidemment, l'œil clos, la forêt de bambous et la présence du chien renvoient au pilote de Lost dans une forme réflexive qui invite à réexaminer la série. Plus encore, la jonction du temps marque un horizon indépassable pour une fiction qui s'est bâtie sur deux lignes temporelles. Les lignes du temps ne semblent pouvoir se rejoindre que dans la fin de la fiction. Alors que Jack glisse lentement vers sa fin, l'apparition finale de l'avion quittant l'île métaphorise le départ de Jack vers la mort, le départ tant convoité au fil de la série et l'abandon de l'univers spécifique qui a permis à la crise de perdurer. 
Figures 3 et 4 : Jack assiste au départ de l'avion dans les derniers instants de la série
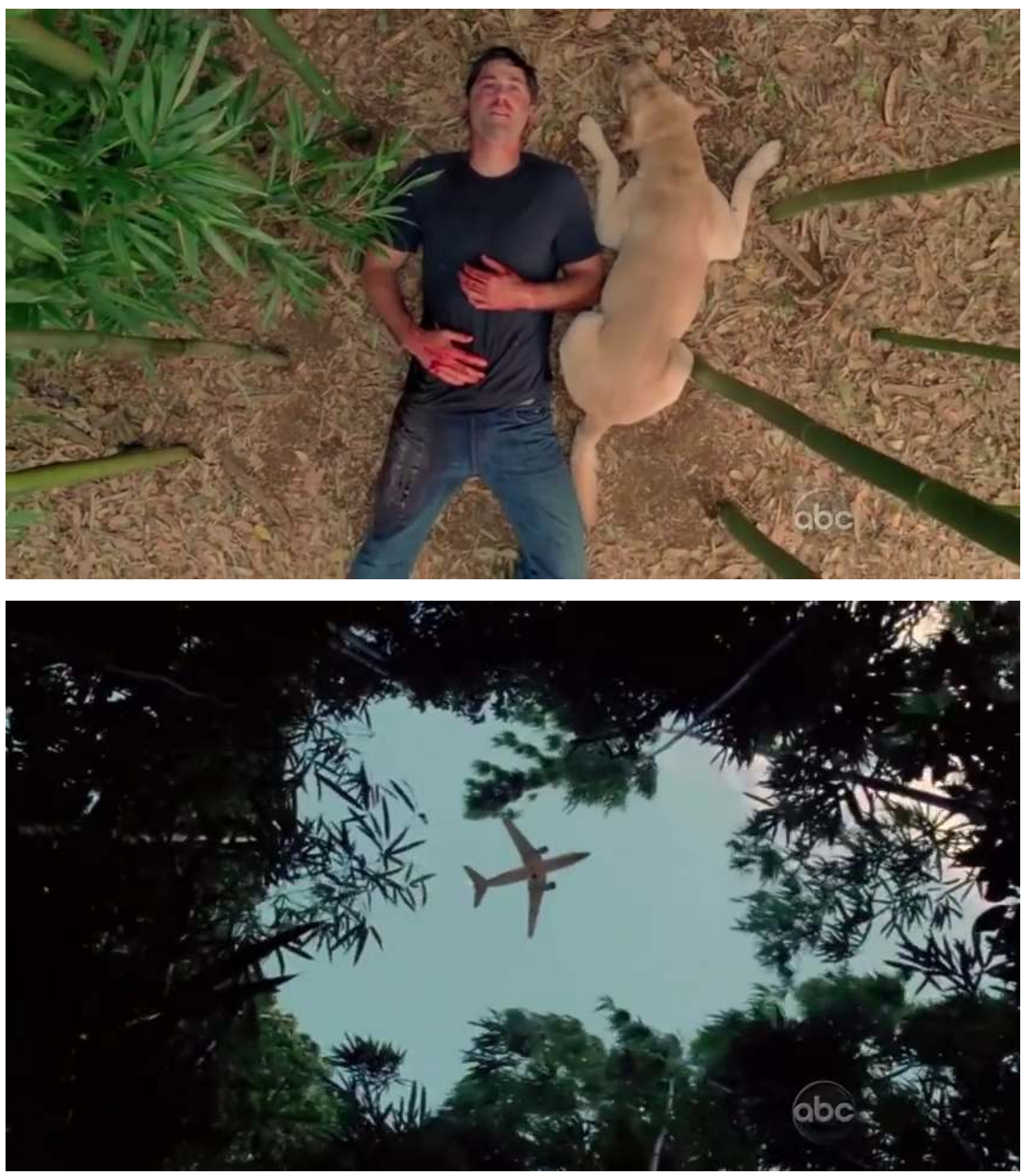

13 La mise en scène gravite autour de Jack : la caméra vise la frontalité du point de vue du personnage, mais avant cela, elle tourne autour de lui, toujours légèrement désaxée par rapport à son regard, avant de lui faire pleinement face lors du tout dernier plan. L'œil qui se ferme ne scrute plus le temps, il scrute les téléspectateurs et marque la dernière jonction inédite de Lost, celle du temps du personnage avec le temps des spectateurs. La mise en scène attend précisément la fin de la séquence pour faire face au regard de Jack comme si l'objectif venait repérer, dans le regard, quelque chose d'unique. À l'instar de Pacôme Thiellement, il nous semble que "La spécifié de Lost, c'est de nous avoir montré que regarder, ce n'est pas seulement subir ; regarder, c'est voir; et voir, c'est être ${ }^{9}$. " Le dispositif visuel tend à révéler que nous ne subissons plus l'action de la fiction, à l'instar de Jack. Le regard constitue l'affirmation de notre identité.

14 La mort de Jack est symbolisée par un fondu au blanc qui renvoie au mode opératoire des morts dans Six Feet Under (Alan Ball, HBO, 2001-2005). Ce n'est pas la première fois qu'un fondu au blanc est utilisé dans Lost. Il y a eu, en fait, trois occurrences. La première correspond à l'implosion de la station du Cygne à la fin de la seconde saison, dont les 
conséquences ne sont dévoilées qu'au début de la troisième saison. La seconde marque une perturbation du continuum temporel avec la mise en mouvement de l'île par Ben, à la fois dans l'espace et dans le temps. La troisième est l'implosion de l'ogive nucléaire par Juliette à la toute fin de la cinquième saison. Chacun de ces fondus est la marque d'une modification du statut de l'île (mise à mal du champ magnétique, déplacement de l'île dans l'espace et dans le temps, création - apparente - d'une autre ligne temporelle). Or, dans cette séquence finale, le fondu au blanc ne sert plus le même but. Il est une marque de la jonction des deux temporalités. Lorsque le fondu au blanc efface les flash-sideways, l'œil de Jack se referme. Cette lumière blanche affirme l'imminence de la mort de Jack, mais celle-ci a lieu dans la sérénité. À l'inverse des autres utilisations du fondu au blanc, celui-ci n'est pas le signe d'une perturbation, mais d'une réunification temporelle apaisée. Jack voit au-delà de sa propre temporalité et cette vision a du sens pour lui. Dans les derniers plans de la séquence, le travelling arrière où la caméra s'écarte de Jack laisse place à un contre-champ qui révèle le départ de certains survivants en avion. Si un travelling similaire marquait, dans le pilote, la présence de Jack sur l'île, ce dernier mouvement de caméra affirme son départ. La quête initiale de Jack trouve une forme d'accomplissement puisque le départ de l'île est au cœur des récits de Lost.

\section{Perturbation du schème sensori-moteur}

Dans Lost, la jonction du temps n'est rendu possible que par une perturbation du schème sensori-moteur, c'est-à-dire de notre capacité à nous mouvoir et à percevoir notre environnement. La mise en scène glisse d'une image-mouvement à une image-temps selon la pensée de Gilles Deleuze. L'image-mouvement repose sur une relation de causalité, un rapport de situation-perception-action (exemple : j'ai soif, j'ai une bouteille devant moi, je prends la bouteille pour boire). Dans la dernière séquence, la blessure grave de Jack perturbe son schème sensori-moteur. Il peut encore sentir mais de moins en moins bouger. L'image tend alors à dévoiler des situations optiques et sonores pures, se rapprochant sensiblement de l'image-temps au sens de Deleuze. La mise en scène glisse donc d'un dispositif audiovisuel fondé sur l'image-mouvement à un dispositif plus ambigu, où nous ne sommes plus dans l'image-mouvement sans être encore pleinement dans l'image-temps. Comme le rappelle Deleuze, le postulat d'un monde unique vole en éclats avec l'image-temps. Cette séquence n'est pourtant pas une image-temps "pure " dans la mesure où le montage induit une forme indirecte de perspective du temps, mais la frontière image-temps/image-mouvement est bel et bien brouillée.

L'image-temps semble présupposer une absence de montage par le plan-séquence, mais ce n'est pas nécessairement aussi tranché car, comme l'énonce Deleuze :

[L] e montage reste le plus souvent l'acte cinématographique essentiel. Seulement, il change de sens : au lieu de composer les images-mouvement de telle manière qu'en sorte une image indirecte du temps, il décompose les rapports dans une image-temps directe de telle manière qu'en sortent tous les mouvements possibles ${ }^{10}$.

La fin de Lost tente de faire sortir les mouvements du temps qui traversent la fiction. Jack est cantonné à une expérience visuelle pure qui s'intensifie au fur et à mesure que son corps s'éteint. Lost nous invite à penser le rapport au temps à travers la mise en scène. En brouillant les frontières de l'image-mouvement et l'image-temps, la série prouve sa 
capacité à se réapproprier des propriétés du cinéma pour les intégrer à son propre discours et à son dispositif.

\section{Discours intemporel}

18 La jonction du temps sert la déformulation du récit puisque cette jonction est inédite dans la série. Il faut d'ailleurs noter l'apparition muette du titre «LOST » après la dernière séquence - précédemment, ce titre était accompagné d'un grondement sonore. L'apaisement amorcé dans la séquence est ainsi poursuivi comme si la tension qui traversait le programme s'était soudainement dissipée. L'épisode série rompt jusqu'au bout avec la formule habituelle de la série.

Comme nous l'évoquions au début de notre propos, nous avons tous notre manière de regarder Lost, mais ces différences peuvent cacher un socle commun. Nous apprécions ces personnages et nous entretenons avec eux un rapport de mémoire particulier, ce que Jean-Pierre Esquenazi appelle mediacy, l'héritage médiatique, ces "savoirs acquis concernant la télévision ${ }^{11} »$. Nous pourrions élargir le sens de ce terme à nos savoirs acquis tout au long du visionnage d'une fiction inscrite dans ce médium. Peut-être que certains d'entre nous se souviennent davantage des flashbacks de Hurley ou de ceux de Sawyer, mais nous partageons tous une sorte de mémoire collective.

La problématique de la fin d'une série nous semble triple: contenter les auteurs/ scénaristes, les directeurs de chaîne et les téléspectateurs. De plus, en raison des nouveaux marchés économiques, il faut penser à l'après-diffusion (vente de DVD, Blu-ray, etc.). Il faut réussir la transition entre ce que nous appelons le temps de la première diffusion et celui de la réappropriation multi-médiatique. En cela, la fin joue un rôle pivot, un point de passage qui peut donner envie ou non de se réapproprier l'œuvre. La déformulation choisie par les auteurs de Lost nous semble anticiper et faciliter ces réappropriations multi-médiatiques. Dans la dernière séquence, les auteurs ont fait le choix de donner une place importante aux téléspectateurs. Le discours de Christian Shephard théorise le rapport entretenu avec la fiction et repose sur une forme de remerciements de la part des auteurs à destination du public. D'autre part, la séquence fonctionne sur une réactivation des affects de la série. Les retrouvailles sont d'autant plus fortes que certains personnages (Boone, Libby ou même Locke) avaient disparu de la fiction depuis plusieurs saisons. Cette séquence portée par des embrassades et des regards complices convoque l'ensemble de la mémoire de la série. Sans parole, les retrouvailles visent à produire une catharsis au sens aristotélicien, une forme d'épuration des sentiments pour les personnages comme pour les téléspectateurs. Les regards, les accolades, les poignées de mains traduisent la circulation des affects entre les personnages et encouragent à réexaminer sa propre mémoire spectatorielle. Comme le rappelle Shawn Ryan : « Pour moi la marque d'une série qui restera dans les annales de la télé, c'est une fin qui donne envie de réexaminer le début ${ }^{12}$.» Lost apparait comme un parfait exemple puisque la série incite à la remémoration tout en affirmant un discours intemporel.

21 En effet, les flash-sideways s'inscrivent hors de toute temporalité traditionnelle. Il s'agirait peut-être d'un temps toujours présent puisque Desmond peut y accéder de son vivant, mais dont l'objectif final est de connecter les expériences vécues sur l'île - un espace temporel pour se préparer à l'expérience au-delà de la mort mais où il faut d'abord se 
souvenir de la vie. Comme nous l'avons vu, le discours de Christian et les retrouvailles dans l'église tendent à réactiver la mémoire fictionnelle des spectateurs. Lorsque Jack retrouve Boone, c'est ainsi une partie de la première saison qui est convoquée.

La marque la plus évidente qui donne envie de revoir le début est, bien sûr, la mort de Jack qui fait pleinement écho à son réveil dans l'épisode pilote. De cette manière, la fiction fonctionne au travers du regard de Jack qui ouvre et referme la série, comme si, sans ce dernier, il n'y avait plus de fiction possible. Enfin, le jeu de parallélisme inversé entre la fin et le début tend à affirmer une forme de boucle temporelle. Ce renvoi final aux prémices de la série marque le désir des auteurs de faire de leur série une fiction autoréflexive ou un "palimpseste réflexif ${ }^{13}$ ». La série réécrit sa propre histoire, puisque les flash-sideways construisent une narration alternative.

Figures 5 et 6 : Jack ouvre les yeux dans le pilote et les ferme dans l'épisode final
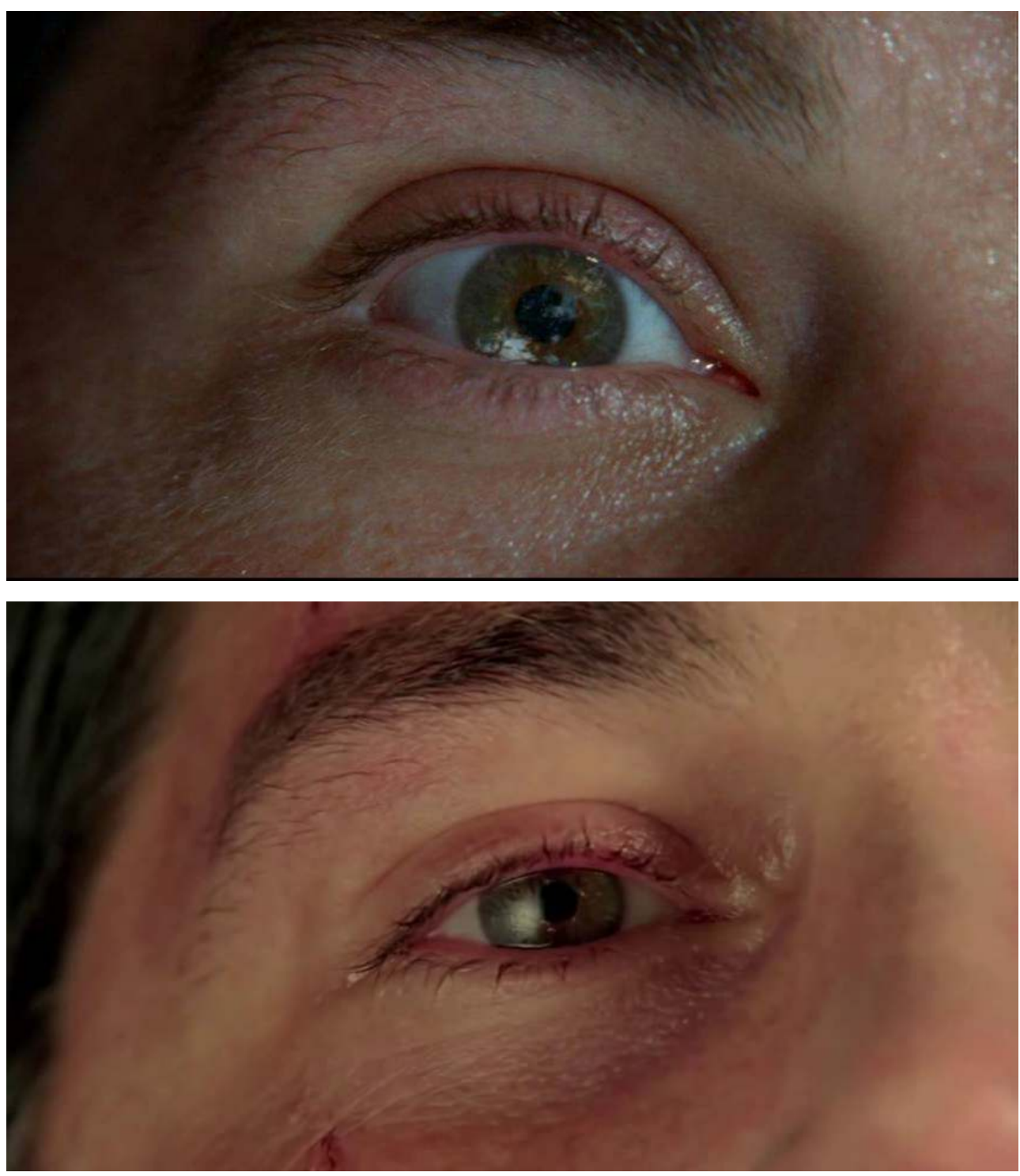

Le discours des auteurs semble donc s'inscrire dans une forme intemporelle. La fin réactive les nombreux affects qui ont façonné la série. Comme l'évoque Christian, ce qui est réel, c'est ce qui a été partagé avec ces personnes. Or, c'est effectivement ce triangle des affects qui inclue à la fois les téléspectateurs, les personnages et les auteurs. Les 
moments de partage entre tous les personnages, mais surtout entre les téléspectateurs et les personnages, font de l'expérience de la fiction une expérience des affects. Ce lieu qu'est la fiction est une œuvre collective dans le sens où les auteurs, avec la collaboration des téléspectateurs, ont pu façonner un monde fictionnel du partage. L'intemporalité du discours de Lost, c'est justement cet espace-temps des flash-sideways, temps purement fictionnel qui déjoue toutes les manières de penser le temps pour faire triompher la fiction sérielle dans ce qu'elle produit de plus spécifique - le triomphe des relations humaines.

La fin marquée par la mort de Jack laisse une ouverture à la continuité de l'univers : l'île continue, en effet, d'exister avec un nouveau gardien. Lost semble proposer une approche quasi cyclique du récit sériel, portée par un regard. Les auteurs privilégient la fin d'une expérience plutôt que la fin d'un univers. Ainsi, l'expérience de Lost s'inscrit dans une intemporalité rendue possible par les modes de regard des téléspectateurs et par l'idée que les affects survivent à la fin de la fiction.

En articulant la fin autour d'une nouvelle dimension temporelle et d'une jonction inédite, Lost affirme la spécificité de son discours temporel. Conscients que les téléspectateurs inscrivent l'expérience de Lost dans un mode de regard spécifique qui induit une appropriation temporelle, les auteurs synthétisent cette idée en lui donnant corps et forme et en proposant de faire des flash-sideways une temporalité de la "téléspectature » où chacun peut investir son propre mode de regard. Le rôle central de Christian incarne la figure de l'auteur qui prend par la main le personnage/spectateur pour lui faire traverser cette expérience du passage de l'immanence vers l'imminence de la fin du récit. Lost crée ainsi les conditions d'un réinvestissement dans la fiction puisque la fin pousse à réexaminer l'ensemble de la série. Comme l'énonce Locke dans l'épisode «Orientation » (S2E03), à travers une formule qui a fait le succès de la série : « We're going to need to watch that again " ("On va devoir regarder ça à nouveau »). La jonction finale du temps, c'est aussi celle de deux temporalités - celle de la fiction et celle des téléspectateurs qui ont répété pendant plusieurs années la même action que Jack, ouvrir et refermer son regard à chaque épisode. Les auteurs tentent de réconcilier deux temporalités incompatibles, la fiction et le réel. Pourtant, à l'instar de Jack qui ne peut quitter son statut physique de personnage de fiction, les spectateurs ne peuvent pénétrer à leur tour physiquement dans la fiction. Le voile donne à voir l'autre temporalité mais empêche de traverser la frontière, seuls les affects semblent franchir la barrière qui sépare la fiction du réel. Si nous reprenons l'idée de "liquidation générale ", Lost a une dette à payer envers les téléspectateurs. Le temps donné à regarder la série doit être rétribué. Comme le rappelle Sarah Hatchuel, «Si les spectateurs ont donné beaucoup de leur temps, de leur vie, de leur énergie (à comprendre, chercher, décoder), la série leur a rendu leur propre vie (peut-être changée, voire transcendée) en retour ${ }^{14}$.»

L'expérience de la fiction met en avant le rôle des affects et la circulation à double sens opérée par ces derniers. Jack se construit finalement à travers les relations qu'il entretient avec les autres personnages. Jack le cartésien traverse la fiction avant de devenir un homme de foi et remplacer Jacob. Il façonne son identité au cours de la série aidé par les affects qu'il partage avec les autres personnages. Dans les dernières minutes de Lost, l'apaisement de Jack semble être concomitant de la double perception temporelle qui l'amène à mettre en perspective le chemin parcouru. Jack peut reposer en paix : il a compris que son expérience n'a de valeur qu'à travers les affects qui l'ont façonné. 

rompt avec la structure temporelle, sonore et esthétique (la photographie de la scène de l'église convoque un éclairage et un étalonnage très doux, uniques dans la série). « Nobody does it alone, Jack» (« personne n'y arrive seul »), pourtant Jack s'écroule seul sur l'île, mais sa solitude est illusoire dans la mesure où il est entouré par les affects. Comme l'analyse Pacôme Thiellement :

$$
\begin{aligned}
& \text { Et, en tant que miroir de la mort, ceux-ci doivent s'y souvenir (de leur } \\
& \text { véritable nature), laisser partir (leurs passions) et partir eux-mêmes dans } \\
& \text { une autre dimension. Mais il est également le miroir de la vie pour le } \\
& \text { spectateur. Et celui-ci doit passer à autre chose (sa propre vie), laisser partir } \\
& \text { (le récit) et se souvenir, à son tour également, de sa nature divine. Cette vie- } \\
& \text { dans-la-mort, c'est notre vie de spectateurs }{ }^{15} \text {. }
\end{aligned}
$$

Christian aurait pu ajouter « Death is not the end » (« La mort n'est pas la fin »), tant la fin de la série ne signifie pas sa mort. Les affects qui l'ont composée sont emportés par les téléspectateurs, assurant ainsi une forme de continuité dans un autre monde. Nous continuons à discuter de Lost, nous continuons à conseiller à des amis de regarder cette fiction, nous ré-expérimentons l'œuvre. Quand nous souhaitions que Lost s'achève rapidement - voire dès sa première saison - nous étions loin d'imaginer à quel point cette question du temps allait donner une telle aura à cette fiction.

\section{BIBLIOGRAPHIE}

DELEUZE Gilles, L'Image-temps, Paris, Les éditions de Minuit, 1985.

ESQUENAZI Jean-Pierre, Les séries télévisées : l'avenir du cinéma ?, Paris, Armand Colin, 2010.

GENETTE Gérard, Discours du récit, Paris, Éditions du Seuil, 1972.

HATCHUEL Sarah, Lost : Fiction Vitale, Paris, Presses universitaires de France, 2013.

KERMODE Frank, The Sense of an Ending, New York, Oxford University Press, 2000.

ODELLO Laura et SZENDY, «Fins de séries », Artpress2 : Séries télévisées formes, fabriques, critiques, février/mars/avril 2014, $\mathrm{n}^{\circ} 32$, p. 36-41.

RICCEUR Paul, Temps et Récit 2. La configuration dans le récit de fiction, Paris, Le Seuil, 1984.

THIELLEMENT Pacôme, Les mêmes yeux que Lost, Clamecy, Éditions Léo Scheer, 2011.

\section{NOTES}

1. Frank Kermode, The Sense of an Ending, New York, Oxford University Press, 2000.

2. Paul Ricœur, Temps et Récit 2. La configuration dans le récit de fiction, Paris, Le Seuil, 1984, p. 49.

3. Jean-Pierre Esquenazi, Les séries télévisées : l'avenir du cinéma ?, Paris, Armand Colin, 2010, p. 26.

4. Laura Odello et Peter Szendy, "Fins de séries", Artpress2 : séries télévisées formes, fabriques, critiques, février/mars/avril 2014, $n^{\circ} 32$, p. 36-41. 
5. Sarah Hatchuel, Lost : Fiction Vitale, Paris, Presses universitaires de France, 2013, p. 124.

6. Gérard Genette, Discours du récit, Paris, Éditions du Seuil, 1972, p. 39.

7. Ibid., p. 66.

8. Nous pouvons aussi noter le baiser échangé entre Juliette et Sawyer qui renvoie directement au baiser échangé lors de la mort de cette dernière (S6E01).

9. Pacôme Thiellement, Les mêmes yeux que Lost, Clamecy, Éditions Léo Scheer, 2011, p. 53.

10. Gilles Deleuze, L'Image-temps, Paris, Les éditions de Minuit, 1985, p. 170.

11. Esquenazi, p. 5.

12. Lost, saison 6, DVD, ABC Studio, 2010, bonus, documentaire, «La création de la dernière saison ".

13. Hatchuel, p. 116.

14. Hatchuel, p. 134.

15. Pacôme Thiellement, p. 113-14.

\section{RÉSUMÉS}

Ce texte propose de s'interroger sur la fin de Lost sous un angle temporel et esthétique. Source de nombreux débats intenses, la conclusion de Lost nous offre une occasion de nous interroger sur notre manière de regarder la série. En nous appuyant sur notre théorie de la déformulation, nous analyserons la manière dont la fiction se défait sous nos yeux tout en réalisant l'impossible jonction de deux lignes temporelles antinomiques. La série établit un lien fort avec le téléspectateur en élaborant un double discours final dont le second destinataire est le téléspectateur. Par un montage final subtil et cohérent, les auteurs de Lost lient nos expériences, les lignes temporelles, les personnages dans un même but, provoquer une catharsis émotionnelle, dont la portée, tend à inscrire la fiction dans la mémoire collective de manière indéfinie. Lost nous réapprend que la fin a de la valeur dans la mesure où nous avons partagé des affects avec autrui. Parce que Lost est une fiction sur des personnages perdus qui, dans les derniers instants de la fiction, se trouvent.

This text offers a questioning on the ending of Lost from a temporal and aesthetic angle. As it has been the source of many strong controversies, the conclusion of Lost gives us an opportunity to wonder about the way we watch the show. Through our deformulation theory, we shall analyze how fiction unravels before us while accomplishing the impossible junction of two antinomic temporal lines. The show sets up a strong connection with the TV viewer as it elaborates a final double-speech whose second listener is the TV viewer. Through a subtle and coherent final cut, the authors of Lost connect our experiences, the temporal lines, and the characters in a same goal: producing an emotional catharsis, whose scope tends to indefinitely engrave the fiction in the collective memory. Lost reminds us that the value of the ending also consists in having shared some affect with someone else, since Lost is a fiction about lost characters who, during the last moments of the fiction, find themselves. 
INDEX

Mots-clés : Lost, déformulation, image-temps, image-mouvement, fin, conclusion, dénouement Keywords : time-image, movement-image, ending, closure

\section{AUTEUR}

\section{VLADIMIR LIFSCHUTZ}

Vladimir Lifschutz a achevé un doctorat en étude cinématographique à l'université Lumière Lyon 2 sous la direction de Jean-Pierre Esquenazi et Martin Barnier. Il travaille sur la question des configurations temporelles dans les séries télévisées, mais aussi sur les clôtures de série, les univers fictionnels et la dimension esthétique et narratologique des œuvres sérielles. Il s'intéresse plus largement aux mutations narratives et à l'histoire des récits. Vladimir Lifschutz completed his thesis in film studies at the University of Lyon 2 under the direction of Jean-Pierre Esquenazi and Martin Barnier. He works on the question of temporal patterns in TV series, on the question of how to end a TV show, and on the fictional universe and the aesthetic and narratological dimensions of fiction. He is also interested in narrative mutations and the history of stories. 